\title{
Feasibility Demonstration of Terrestrial RNP with LDACS
}

\author{
Okuary Osechas ${ }^{1}$, Shrivathsan Narayanan ${ }^{1}$, Omar García Crespillo ${ }^{1}$, Gianluca Zampieri ${ }^{1}$, \\ Giuseppe Battista ${ }^{2}$, Rachit Kumar ${ }^{3}$, Nicolas Schneckenburger ${ }^{4}$, Elisabeth Lay ${ }^{5}$, Boubeker \\ Belabbas $^{1}$, and Michael Meurer ${ }^{1}$ \\ ${ }^{1}$ German Aerospace Center (DLR), Oberpfaffenhofen, Germany \\ ${ }^{2}$ Airbus, Ottobrunn, Germany \\ ${ }^{3}$ Airbus, Bengaluru, India \\ ${ }^{4}$ Hensoldt Sensors, Taufkirchen, Germany \\ ${ }^{5}$ TNG Technology Consulting, Unterföhring, Germany
}

\begin{abstract}
In this paper we discuss the first results from a set of flight experiments. The objective was to show that terrestrial ranging can support aviation services with sufficient accuracy for non-precision approach. The experimental setup is based on using LDACS signals to provide pseudorange measurements. The data analysis reveals that the position solutions are compliant with RNP 1 standards. We also propose the feasibility of RNP 0.3 using LDACS as terrestrial ranging.
\end{abstract}

\section{Introduction}

While many existing systems are capable of supporting current and near-term plans in Alternative Position, Navigation, and Timing (APNT) services, it has become apparent that state-of-the-practice technologies will not support the most demanding Performance-Based Navigation (PBN) services. Specifically, to achieve the most demanding Required Navigation Performance (RNP) types, Distance Measuring Equipment (DME) will not be sufficient [1], so new methods and hardware are needed. The use of the L-Band Digital Aeronautical Communications System (LDACS) for navigation applications has long been proposed as a way of pushing the envelope on terrestrial RNP, in particular moving towards RNP 1 or RNP 0.3 types [2].

Project Alps (Alternative Positioning System) was conceived to demonstrate the feasibility of implementing RNP services on terrestrial ranging, using LDACS. The cusp of the Alps effort was a measurement campaign using dedicated elaborate flight trials, in the summer of 2018, which demonstrated how the navigation performance of LDACS can support RNP 1 and even RNP 0.3. This is the first time that an APNT system, based on terrestrial ranging, supports this level of performance.

\section{Motivation}

With increasing levels of air travel, there is increased demand for airspace capacity, which in turn increases the pressure on Air Traffic Management (ATM) systems to become more efficient. One ingredient to improve efficiency is automation, with ATM happening with less and less human intervention. The main benefit of using RNP services is, precisely, that they support aircraft movement with minimal controller involvement.

This trend in automation was initially enabled by the availability of navigation services that rely on Global Navigation Satellite Systems (GNSS). GNSS-based measurements support both navigation and surveillance services (e.g. ADS-B) with position estimates that have higher accuracy than any other certified system but, more importantly, 
also higher integrity.

In order to support these higher levels of automation ATM systems will require a reversion capability, should the primary (GNSS-based) system ever fail. This will require levels of performance that are currently not achievable with terrestrial navigation aids (NavAids). The development of LDACS as a ranging source has made it plausible to provide APNT with sufficient integrity to meaningfully complement satellite-based navigation. The assumption has been that LDACS-based navigation would support protection levels or containment areas adequate for the most stringent RNP services. Verifying this assumption was the key motivations for the flight experiments in Project Alps.

In this paper we present a quantitative discussion flight experiments in Project Alps. This discussion includes an assessment of ranging errors, position-domain errors and their implications for future APNT systems. These results will strengthen the claim for LDACS as a technology with the potential to support terrestrial ranging with integrity for future APNT applications.

\section{Background}

The establishment of LDACS as a ranging source has opened up new possibilities in developing APNT systems, thanks to its ranging accuracy. The processing of data from the initial flight trials indicated that LDACS would likely support ranging accuracy in the order of $20 \mathrm{~m}$ or better standard deviation 2, 3. A demonstration of LDACSbased positioning, however, had not been achieved at the time of the first trials. The results in this paper are meant to dispel any doubts about the feasibility of LDACS-based positioning, as well as answer many design questions that had not been addressed previously.

A component of APNT that is often left implicit is the objective of supporting Performance-Based Navigation (PBN). More specifically, in Europe there is a desire to support Required Navigation Performance (RNP) services on a wide scale. The distinguishing feature of RNP is that each RNP type has a required maximum Total System Error (TSE) error associated with it, indicated by a number. For navigation purposes, there is a requirement on the Navigation System Error (NSE) associated with each type. The details of how the different types map into NSE bounds are best reviewed in [4, though some discussions on this topic have appeared in previous papers [5], [6]. For the purposes of this paper, two values are critical: RNP 1 requires the $95 \%$ horizontal navigation error to be bounded by $1604 \mathrm{~m}$, while for RNP 0.3 that number is $307 \mathrm{~m}$.

The integrity requirements for RNP services are less stringent than they are for precision approach or landing, yet at the same time, no established methodology exists to prove the $95 \%$ bounds on the position errors. RNP requires that the navigation system provide On-Board Performance Monitoring and Alerting (OBPMA). The exact method by which this capability will be achieved for APNT systems is still to be determined. A variety of proposals exist, ranging from hybridization with inertial systems [7] to increasing the redundancy of terrestrial measurements by hybridizing various navigation technologies [8].

A key point of discussion in the development of LDACS as a terrestrial navigation system has been its relationship to existing navigation aids (NavAids) and systems. Essentially, two possibilities are conceivable: (a) LDACS as an independent, stand-alone system, or (b) LDACS in a hybrid solution with other systems, most notably DME.

Regarding hybridization, we make no assumption in this paper about combining LDACS with other ranging systems. The flight experiments were set up to support an LDACS-only navigation solution. However, recognizing the need for redundancy of measurements, the topic remains on the list of items for future work. The results of this paper are representative of the kind of navigation services that could be derived from a dedicated network of LDACS pseudolites, without taking into account other, established navigation systems. 


\section{Methodology}

The main purposed of the experiments was to demonstrate the ability of an LDACS-based system to provide navigation solutions compliant with RNP 1 or RNP 0.3. For that purpose we implemented four terrestrial LDACS-based ranging sources, as well as a software-based LDACS receiver. We mounted the receiver on a research aircraft and flew the setup in the vicinity of the four ground stations. The details experimental setup will be described in greater detail in the corresponding section.

In our setup station transmits an LDACS signal, synchronized to have the same transmission times as the other stations. The airborne setup is able to record the radio signals in a way that pseudorange measurements can be computed a posteriori. With these measurements we determine LDACS-based position estimates that can be compared with an on-board, GNSS-based truth system.

The key parameter for the experiments is the horizontal position error of the LDACS-based solution. In contrast with precision approach applications, like LPV-200 9] or GAST-D [10, non-precision services like RNP have no requirement for vertical positioning. Vertical guidance is typically provided from barometric measurements and position solutions are typically reported in terms of latitude, longitude and flight level.

Note that the flight level does contain vertical information. However, flight levels are usually defined in terms of atmospheric pressure, rather than geometric distance, and so a vertical navigation error is not defined. The inability of an aircraft to adhere to a flight level is not a matter of navigation but of flight controls. As such, any uncertainty in vertical positioning out to be accounted for in the flight technical error (FTE), rather than the NSE.

As currently envisioned, LDACS will be a pseudoranging system. While there are many benefits of pseudoranging over two-way ranging, one of the limitations is that the observability of the user clock bias degrades outside the footprint of the ground stations. This reflects in the dilution of precision (DOP) degrading quickly upon exiting the footprint (see Fig. 6). Translated into an operational scenario this fact can be translated into a requirement for siting ground stations appropriately, especially on the boundaries of the service volume.

To emulate the performance of a real-world system, all the stored data is processed offline. Some of the processing is not real-time like, as it requires forwards-backwards processing. GNSS-free synchronization of transmitter clocks can be achieved with known algorithms, but would require an assured real-time data channel, which is out of scope of the experiment and remains an item for future work. In an experimental context it is justifiable to focus efforts on designing the signal processing and navigation solution, on the airborne side, while assuming that a feasible solution for real-time synchronization exists.

The a-posteriori synchronization is realized by providing each ground station with an accurate, GNSS-based time reference. We then compensate for clock behavior in an a-causal fashion. In a deployment scenario, synchronization would not be based on GNSS signals, which was beyond the scope for the experiments as discussed. The on-board signal processing and positioning algorithms, however, are implemented in a way that they are compatible with real-time operations.

For the computation of pseudorange observables the processing consists of correlating the incoming signal with the known parts of the LDACS wave form. The resulting TDOA measurements are then smoothed using their Doppler signature. The details of the synchronization setup and the signal processing can be consulted in [2].

\section{Experimental Setup}

The experimental setup for the test campaign consisted of four ground stations and the airborne equipment. For the purposes of demonstrating the feasibility of LDACS-based pseudoranging, it was sufficient to use high-end prototyping hardware (like arbitrary wave-form generators and radio-frequency recorders), as opposed to developing specialized hardware. As will be seen in the discussion of the results, the performance of such prototyping hardware is sufficient to support RNP-type services. The development of dedicated synchronization hardware is out of scope 
for this set of experiments.

\section{Ground Stations}

We placed four ground stations within $60 \mathrm{~km}$ of each other. The main site was set at the Oberpfaffenhofen airport, for logistical reasons. The remaining sites were chosen for having unobstructed view of the area inside the footprint of the four stations.

$\begin{array}{lll}\text { Oberpfaffenhofen (OP) } & 11.2778^{\circ} \mathrm{N} & 48.0848^{\circ} \mathrm{E} \\ \text { Königsdorf (KD) } & 11.4693^{\circ} \mathrm{N} & 47.8289^{\circ} \mathrm{E} \\ \text { Schwabmünchen (SM) } & 10.7091^{\circ} \mathrm{N} & 48.1801^{\circ} \mathrm{E} \\ \text { Lechbruck (LB) } & 10.8170^{\circ} \mathrm{N} & 47.7122^{\circ} \mathrm{E}\end{array}$

Table 1: Locations of the four pseudolites.

The hardware at the ground stations mainly consists of an arbitrary wave-form generator, along with peripheral hardware designed to ensure synchronization between ground stations. Among other things, the setup provides accurate timing for the transmitted signals.

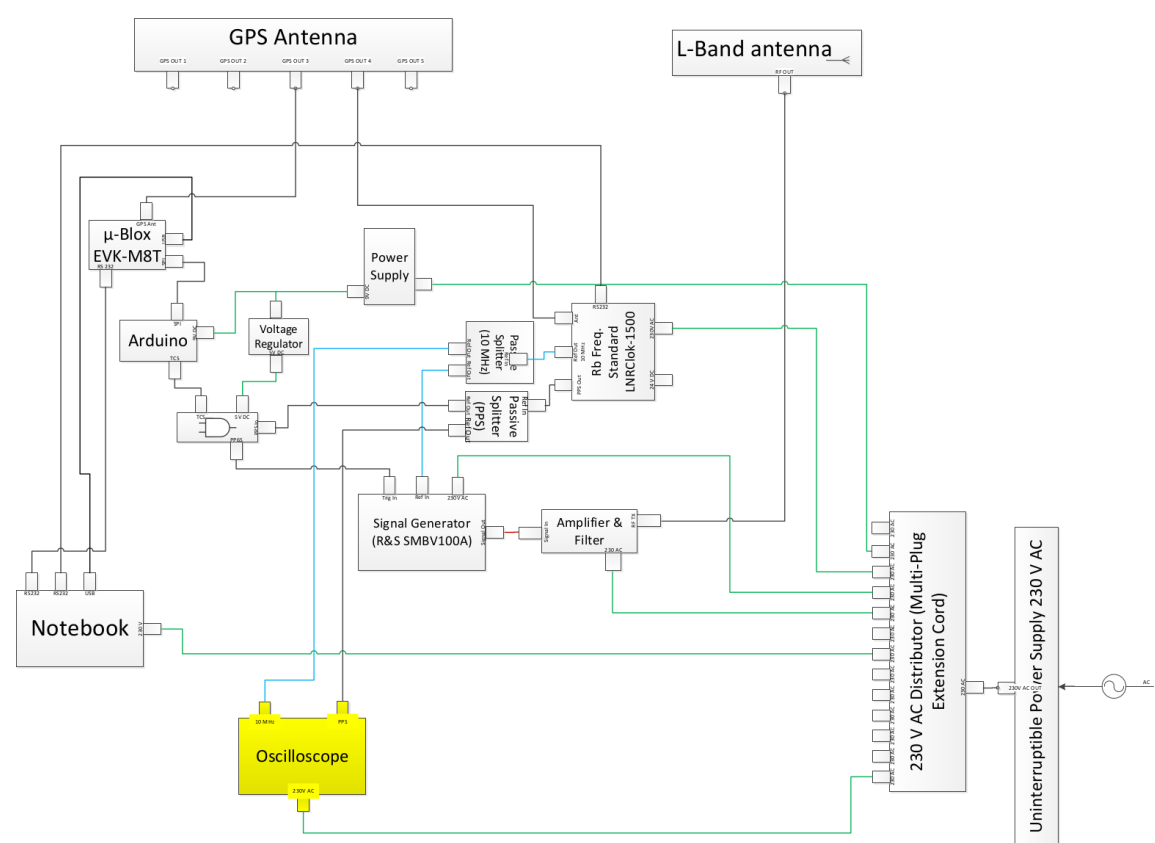

(a)

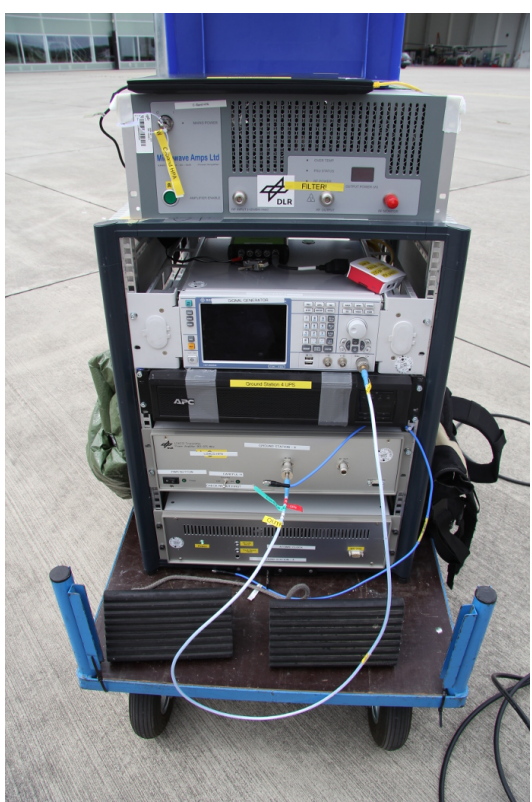

(b)

Figure 1: The central piece of each ground station is the signal generator. There is peripheral hardware to ensure time synchronization between stations and logging of transmit times. (a) Shows the individual modules that make up a ground station. (b) Shows the physical realization of the ground station at Oberpfaffenhofen.

\section{Airborne Equipment}

The hardware was installed on a Falcon 20E, D-CMET, owned and operated by DLR. This aircraft is a modified business jet, adapted accommodate to various research mission. Its most frequent application is in atmospheric and meteorological reseearch, but there are several prior experiences with LDACS measurement flights [11].

The central piece in the airborne equipment consists of a National Instruments PXI I/Q Recorder, peripheral hardware for data logging and time stamping, as well as an air-data system that provides barometric altitude readings. 


$\begin{array}{ll}\text { Atomic Clock } & \text { SpectraTime SRO-100 } \\ \text { Vector Signal Generator } & \text { Rohde \& Schwarz SMBV100A } \\ \text { GNSS Receiver } & \text { Septentrio PolaRX 4 } \\ \text { GNSS Receiver } & \text { u-Blox M8 } \\ \text { High-Power Amplifier } & \text { Custom build from IFN } \\ \text { Transmit L-Band Antenna } & \text { RAMI AV74 DME Broadband Antenna } \\ \text { GNSS Antenna } & \text { Javad AirAnt G3 }\end{array}$

Table 2: List of components of each ground station.

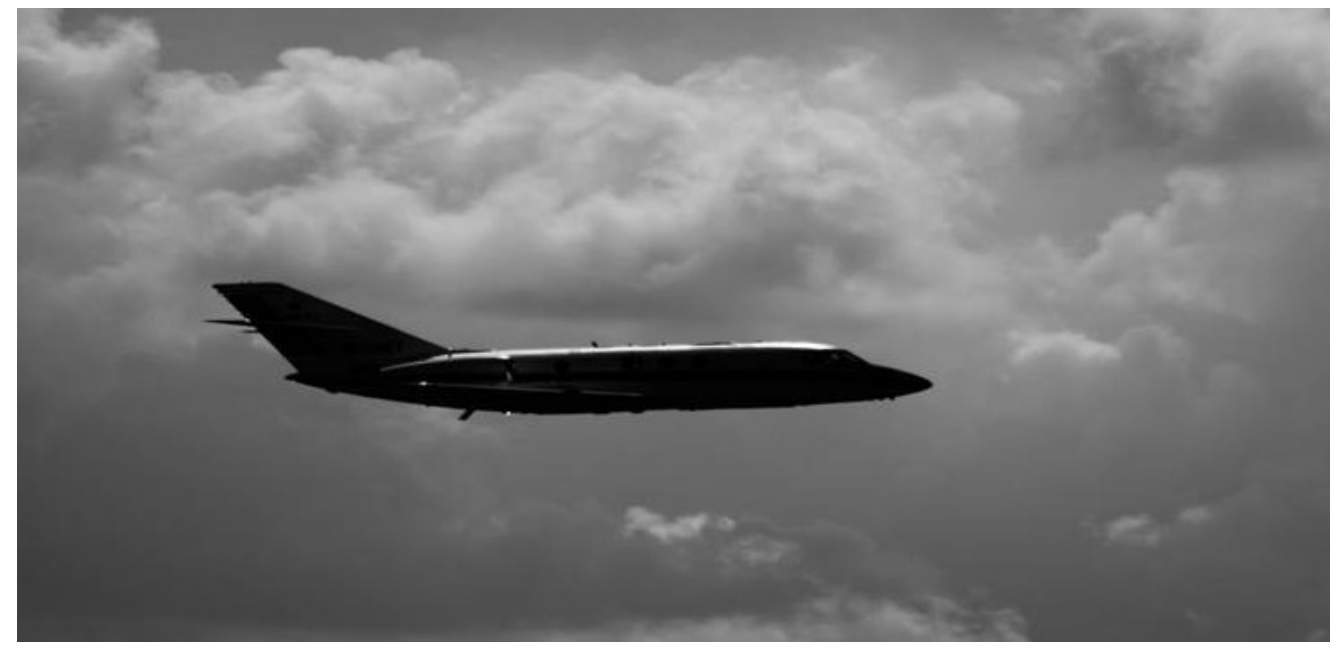

Figure 2: The experimental aircraft (D-CMET) used to collect the data is a modified Falcon 20E.

The truth system is based on a Septentrio PolaRX-2 high-end GNSS receiver that records measurements for postprocessing. In addition, there were two experimental inertial platforms on board, which will help answer a variety of questions in future research.

\section{Trajectory Design}

The flight patterns for the experiments were designed to have as much time within the footprint of the ground stations as possible. The airspace around Oberpfaffenhofen airport (OBI/EDMO) has a variety of constraints, including a major international airport (MUC/EDDM), a traffic restricted area (TRA) for military use and an international boundary with Austria. In practice that means flight trajectories cannot simply be chosen at will, but require close coordination with the Air Navigation Service Provider (ANSP) and are subject to change at short notice. Another consequence is that a significant part of flying time is spent outside the footprint of the LDACS ground stations, which means that not all measurement data can be processed into meaningful position estimates.

For the purposes of modeling position-domain errors we only consider data collected inside the footprint of the ground stations. This is in line with the concept of operations for LDACS as a pseudoranging system, as discussed in [5] and [12. For modeling range-domain errors, this consideration is different, as geometry does not play a role in the collection of the pseudorange measurements themselves, only in the computation of position. In this sense, the data collected outside the footprint will be useful for further analysis, for example in studying ranging errors due to propagation effects. 


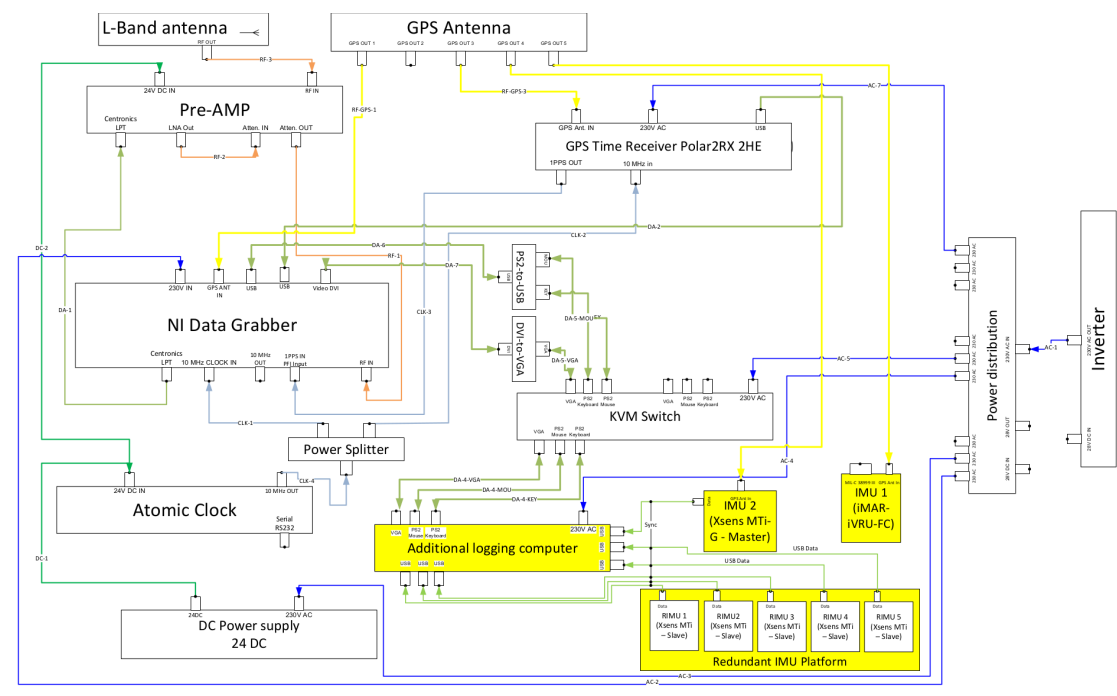

(a)

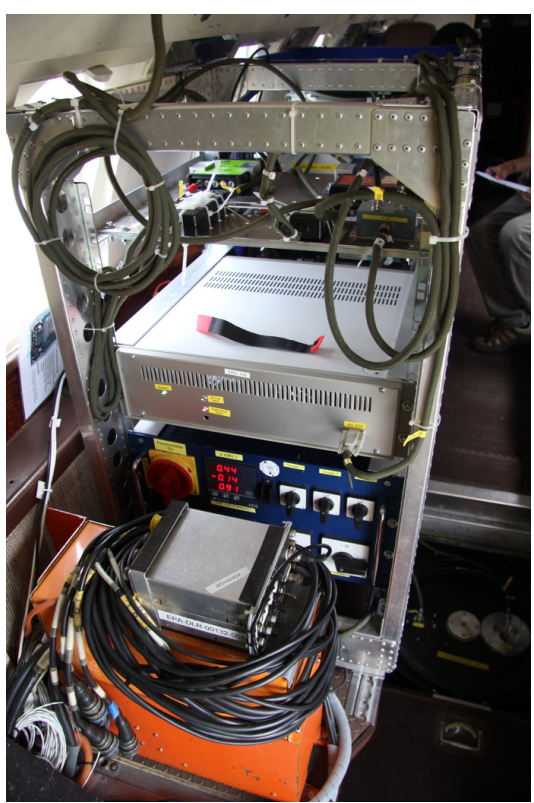

(b)

Figure 3: The airborne hardware is composed of many modules. (a) Shows a schematic diagram of the interconnection between elements. (b) Shows the physical realization as installed in the aircraft, with the power amplifier in the foreground.

\section{Data Processing}

The experiments are set up such that processing happens in two steps: measurement computation and position estimation. The measurements are determined from the radio signals, as sampled by the on-board data grabber. The output of this stage are the pseudorange observables, which are the input for the position estimation process.

\section{Measurement Processing}

As discussed in the Methodology section, data were collected in a way that pseudorange measurements could be computed in software post-processing. On the airborne side, received signals were sampled as in-phase and quadrature (I/Q) components at $20 \mathrm{MHz}$. We process the sampled I/Q values using a software-defined LDACS ranging receiver implemented in Matlab as a maximum likelihood estimator for delay and phase, for details see [3, 2].

The resulting pseudorange observables contain a variety of effects that can be compensated for in another processing step. The plots in Fig. 4 show how biases are compensated in two steps: first we remove calibration biases (e.g. cable lengths), then we remove the clock synchronization biases. In order to verify the ranging capability we compare the distances between the aircraft and ground stations, on the one hand as measured by each LDACS link, on the other, as estimated from GNSS-based positioning.

A striking feature of the plots in Fig. 4 is the number of error values that are many times greater than the standard deviation of the error signal. A majority of these outliers occur outside the footprint of the ground stations and can be explained by low signal-to-noise ratios, as can occur at low elevation angles or when the aircraft banks away from the corresponding ground station. Measurements outside the footprint are beyond the scope of this paper (see Methodology), so we postpone the analysis to future work.

Some of the outliers do lie within the footprint and their impact will be discussed in the section on position-domain errors.

One particular type of outlier that we remove in post-processing are implausible pseudorange readings, in the order 

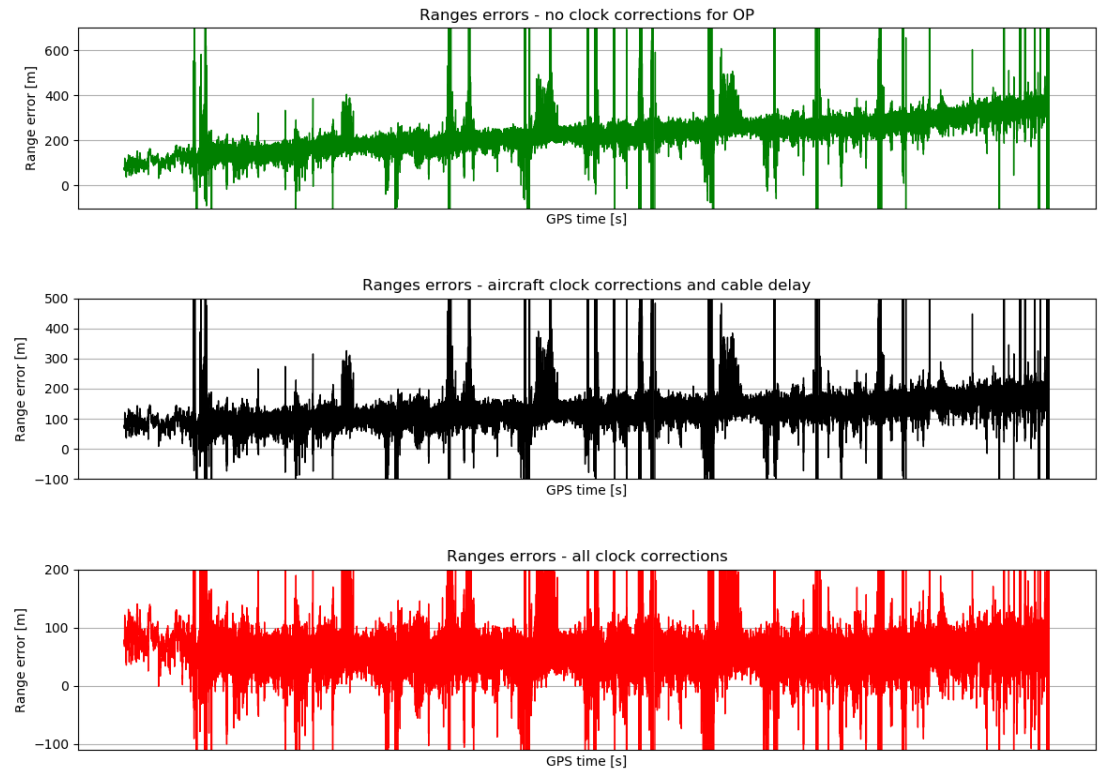

Figure 4: Three processing stages of pseudorange observables. Top: raw pseudorange measurements from station OP. Middle: after compensating for ground-station cable delays. Bottom: After compensating for transmitter clock drift.

of $10^{9} \mathrm{~m}$. While we further investigate the nature of these errors we simply screen out these values, as a measurement of $10^{9} \mathrm{~m}$ is evidently faulted because our aircraft did not fly $1000 \mathrm{~km}$ from any ground station.

There are other anomalies that disrupt the quality of the pseudorange measurements. They include drifting clock anomalies at the transmit stations, during the acquisition phases, as well as as-yet-unexplained spikes in the pseudorange measurements. These spikes result in a structured type of noise that is likely an artifact of the signal processing done for the communications side of the LDACS link. For now these effects have not been understood and remain in the data. These types of outliers do impact the final position-domain error, but we will see that they can be tolerated in the scope of RNP 0.3 operations.

\section{Position Estimation}

The computation of position estimates takes the four pseudorange measurements and the barometric measurement as inputs, to yield a latitude and longitude estimate. In principle, having five measurements to estimate two states would mean that three measurements are redundant, however, in the setup we co-estimate the airborne clock bias, as well as the aircraft altitude, leaving only one measurement for redundancy.

For position estimation we implement an algorithm that is largely based on [13, as cited by [14. Here the barometric measurement provides a constraint for the aircraft to be on an isobaric surface above the ground, minimizing the residuals of the radioelectric pseudorange measurements on that surface. 


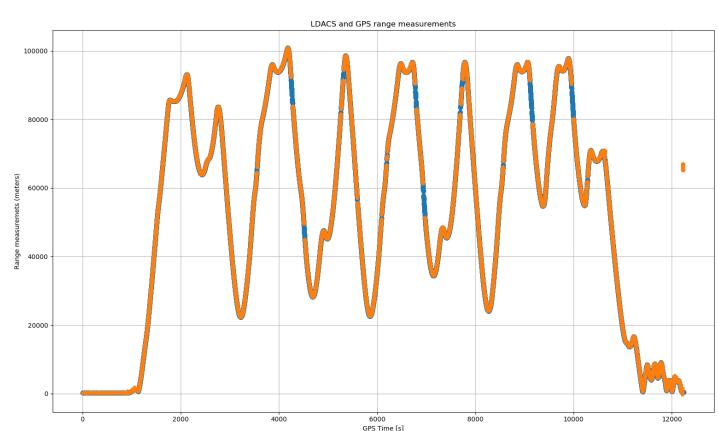

(a)

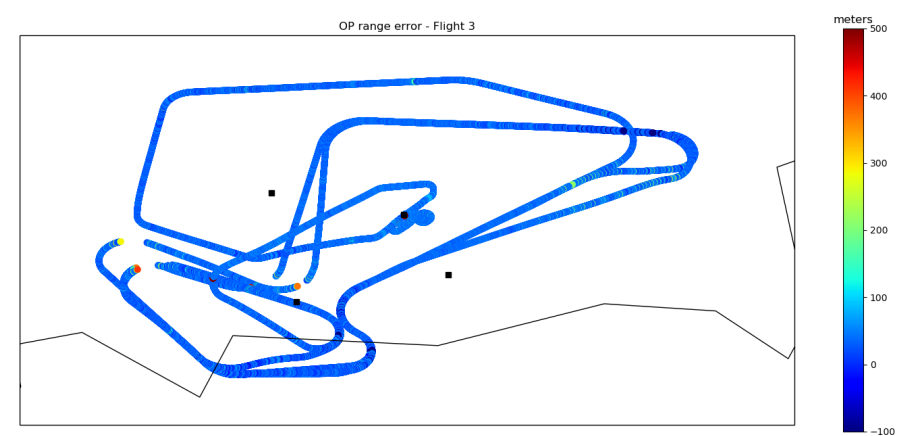

(b)

Figure 5: Validation of LDACS-based pseudorange measurements. (a) Shows how the distance between aircraft and ground station is measure by LDACS (orange) and what the GNSS-based ground truth is (blue). (b) Shows the pseudorange error (LDACS measurement minus GNSS estimate) mapped onto the ground track of one of the flights.

\section{Results}

As described above, we compute position estimates using a Gauss-Newton iterative estimator. We compare the LDACS-based position estimate with a post-processed GNSS-based position fix to compute position-domain errors.

Knowing that LDACS is a pseudorange system, we focus the error analysis to locations that are within the footprint of the four LDACS transmitters, as the DOP becomes unmanageably large on the outside. To illustrate this point we show the dilution of precision (DOP) of the measurements for one flight in Fig. 6. We also indicate the positions of the ground stations and point to the fact that inside the footprint we see DOP values below 3 .

\section{Range-Domain Errors}

In order to give a valid perdiction of how LDACS might perform in a deployment situation, we pre-process the pseudorange errors of Fig. 7. The resulting ranging errors have a negligible mean value and their variability is comparable with previous experimental setups.

\section{Position-Domain Errors}

In Fig. 8 (a) we see that the horizontal error clearly exhibits some strong fluctuations. In the better cases the error behavior appears steady and without major disruptions, except for some structured noise. There are, however, a significant portion of the data with strong outliers. The nature of these outliers is still to be determined, but their existence is not unexpected. It is known that multipath propagation can severely impact terrestrial ranging measurements [15].

It is also known that multipath-related errors have a strong spatial correlation. This correlation stems from the fact that the antennas are fixed in space and most environmental features are, too. Studying the exact origin of the ranging errors that lead to these large position-domain errors remains an intem for future work. However, screening out "faulted" situations from the position-domain error plot, we find that the position-domain error has a 95th percentile of $171.1 \mathrm{~m}$, as pictured in Fig. 9 .

\section{Discussion}

The results of Figs. 7 and 8 suggest that an adequate real-time implementation of LDACS will likely support RNP 1 and even RNP 0.3. It is important to remember that the results come from raw measurements, where appropriate 


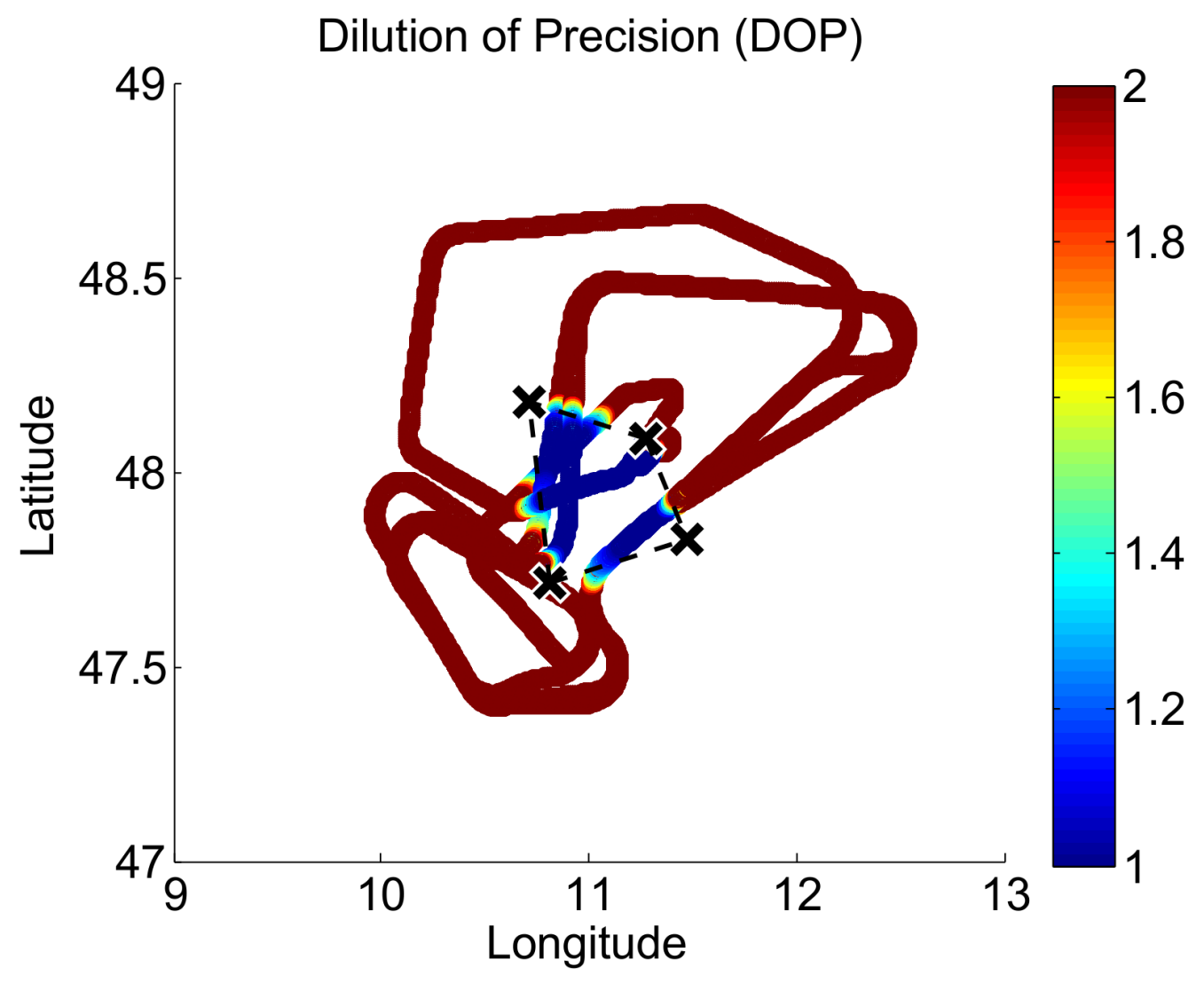

Figure 6: For pseudoranging systems the value of DOP is much lower inside the footprint of the ground stations than outside. For the purposes of this paper we limit the analysis of position-domain errors to DOP $\leq 2$. The plot shows the values of DOP along the flight path for the third day of flying, where values greater than 2 are in red, to better illustrate the point.

smoothing techniques would likely reduce the variability of the signals. The design and implementation of a smoothing strategy remains an item for future work, but given the drift-free nature of the ranging errors in Fig. 7, cautious optimism is warranted.

The accuracy of the pseudorange measurements also suggests that it will be possible to exploit sensor redundancies for On-Board Performance Monitoring and Alerting (OBPMA). However, no specific standard exists for its implementation in terrestrial systems and it is not proven that GNSS-based integrity methodologies apply.

No terrestrial system, currently certified for aviation, supports RNP services. The significance of the results lies in showing the viability of a system that can support RNP during an outage of GNSS-based services.

\section{Summary, Further Work}

This paper gives an overview of the main results from a flight campaign in the Summer of 2018. The original objective was to demonstrate the feasibility of LDACS-based navigation at a performance level of RNP 1 or RNP 0.3. Initial results indicate that the experiments achieved their objective. There are, however, some effects to be understood that, if adequately modeled, could further improve the positioning performance.

On the path to a full RNP capability several issues remain open for further work, among them the treatment of positioning errors due to propagation effects, like multipath or troposphere. The clock anomalies, discussed in the 


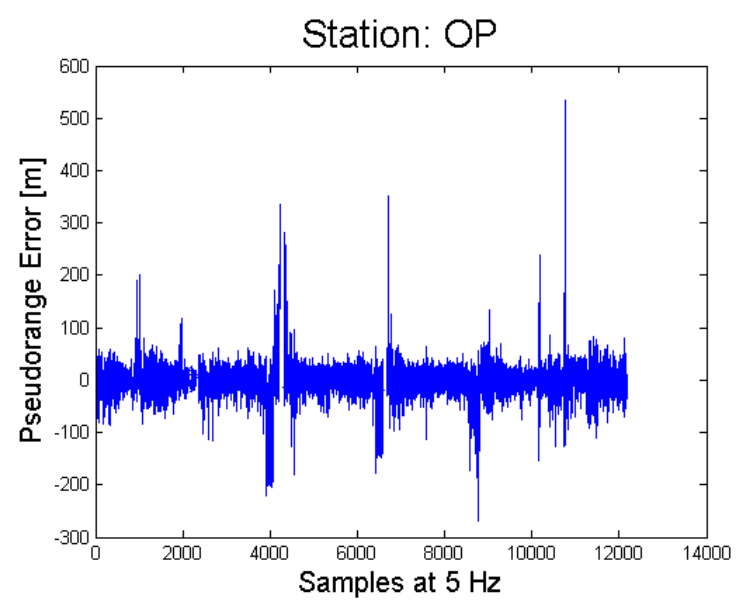

(a)

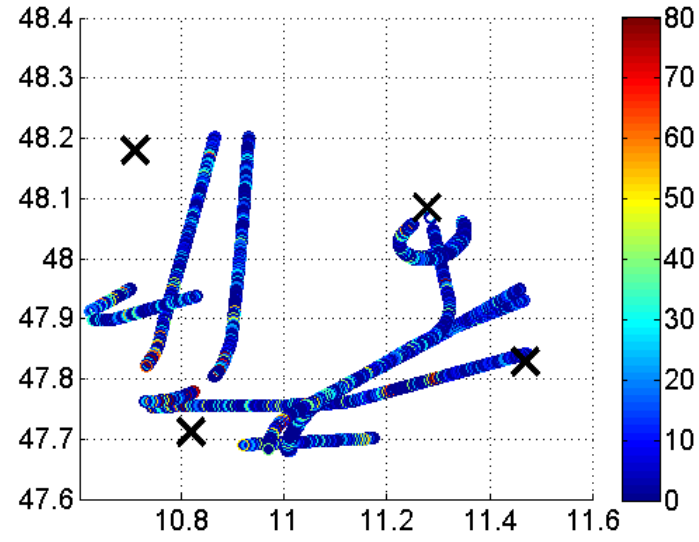

(b)

Figure 7: The pseudorange errors of Fig. 7 need to be pre-processed, to remove measurements outside the station footprint, as well as to cleanse clock transients and other anomalies. (a) Adequately processed pseudorange measurements can reach errors with standard deviations of around $20 \mathrm{~m}$. (b) For the corresponding position errors, $95 \%$ of the samples are below $40 \mathrm{~m}$.

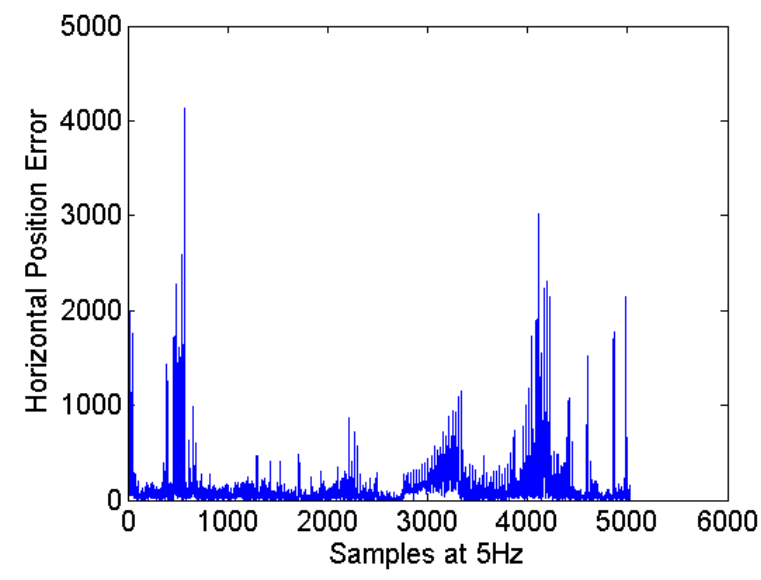

(a)

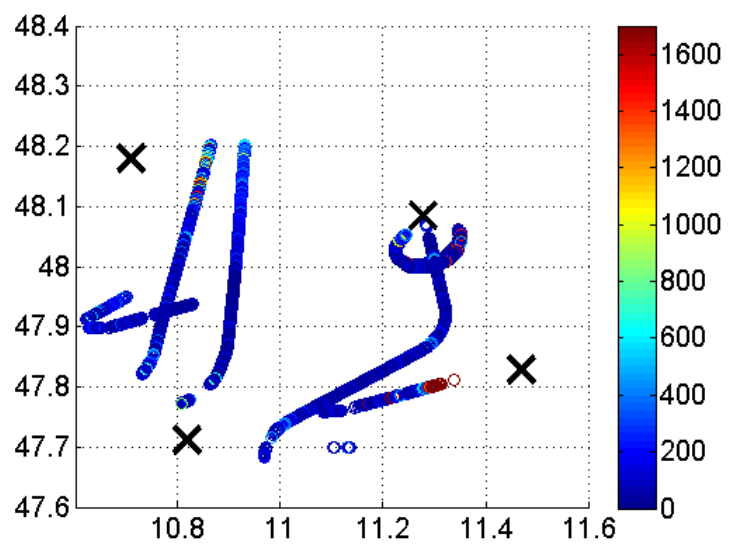

(b)

Figure 8: The pseudorange errors of Fig. 7 need to be pre-processed, to remove measurements outside the station footprint. (a) The position-domain errors in this experiment campaign have a 95th percentile of $495.6 \mathrm{~m}$. (b) There is a strong spatial correlation to position-domain errors.

section on data processing, as well as signal artifacts from the communications side still need to be studied in depth. The most immediate item, however, is to design adequate smoothing for the pseudorange measurements, so as to reduce the position errors further, with the aim of demonstrating RNP 0.3 compatibility.

\section{Acknowledgements}

The authors want to thank the colleagues at DLR, who supported the logistics of the measurement campaign, in particular: Daniel Gerbeth, Simona Cîrciu, María Caamaño Albuerne, Chen Zhu, Nils Mäurer, Dennis Becker and Markus Rippl. We thank the organizations that hosted ground stations: the glider clubs at Schwabmünchen and Königsdorf, as well as Via Claudia Camping. We also want to thank Thomas Richter, of Rohde \& Schwarz, for his support in securing critical hardware for the ground stations. 


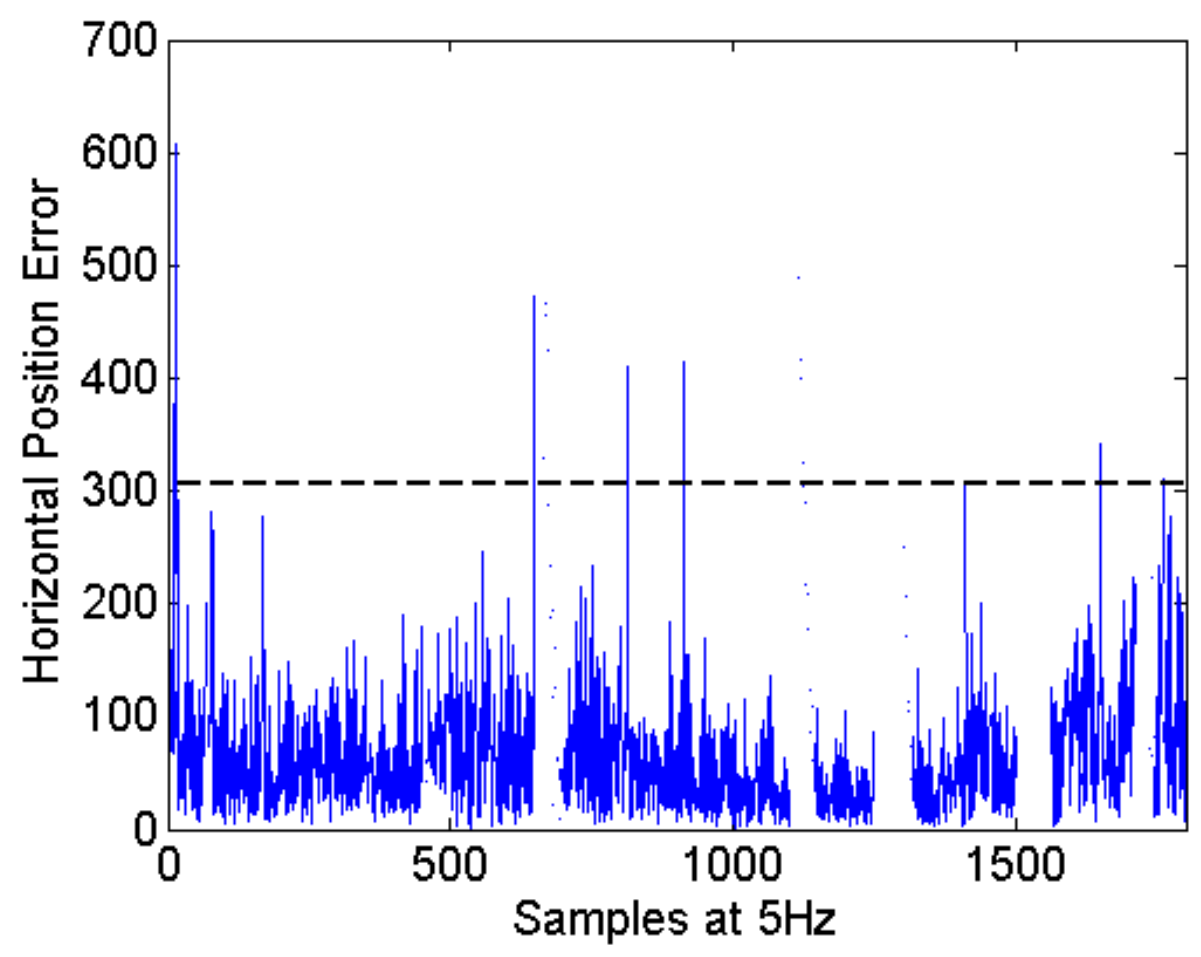

Figure 9: A long stretch of measurement data without faults indicates that RNP 0.3 is feasible with LDACS, though many questions remain to be answered.

\section{References}

[1] G. Berz, V. Vitan, I. Skyrda, and B. Ober. Can Current DME Support PBN Operations with Integrity? In Proceedings of the 26th International Technical Meeting of the ION Satellite Division, ION GNSS+, 2013.

[2] Nicolas Schneckenburger and Bernd Elwischger and Boubeker Belabbas and Dmitry Shutin and Simona Cîrciu and Matthias Süss and Michael Schnell and Johann Furthner and Michael Meurer. LDACS1 Navigation Performance Assessment by Flight Trials. In European Navigation Conference (ENC). Vienna, Austria. 2013. IEEE, 2013.

[3] Thanawat Thiasiriphet, Nicolas Schneckenburger, and Michael Schnell. Ranging With LDACS: Results From Measurement Campaign. In 2016 Integrated Communications Navigation and Surveillance (ICNS), pages 1-18, April 2016.

[4] ICAO. Manual on Required Navigation Performance (RNP), second edition, 1999.

[5] Giuseppe Battista, Rachit Kumar, Elisabeth Nossek, and Okuary Osechas. Placing LDACS-Based Ranging Sources for Robust RNP 1.0 Accuracy En-Route. In Proceedings of the 36th Digital Avionics Systems Conference (DASC), 2016.

[6] Okuary Osechas and Gerhard Berz. Oceanic APNT Using Air-to-Air Ranging. In Proc. of the International Technical Meeting of the ION. Reston, VA, 2018.

[7] Omar García Crespillo, Anja Grosch, and Michael Meurer. Detection of DME Ranging Faults With INS Coupling. In Integrated Communications, Navigation and Surveilance Conference (ICNS). Herndon, VA. $201 \%$. IEEE, 2017.

[8] Okuary Osechas, Elisabeth Nossek, Boubeker Belabbas, and Michael Meurer. A Modular Approach to Integrity for APNT. In Proc. of the Global Nav. Sat. Systems Conf. of the ION. Portland, OR, 2016. 
[9] RTCA. DO-229D: Minimum Operational Performance Standards for Global Positioning System/Satellite-Based Augmentation System Airborne Equipment. Technical report, RTCA, 122016.

[10] RTCA. DO-253C: Minimum Operational Performance Standards for GPS Local Area Augmentation System Airborne Equipment. Technical report, RTCA, 122008.

[11] Thanawat Thiasiriphet, Nicolas Schneckenburger, and Michael Schnell. Impact of the DME interference on the LDACS1 ranging performance. In Proc. of the Global Nav. Sat. Systems Conf. of the ION. Tampa, FL, 2015.

[12] Rachit Kumar, Giuseppe Battista, Okuary Osechas, and Michael Meurer. Optimized placement of LDACS stations to achieve RNP APCH with APNT. In Proc. of the International Technical Meeting of the ION. Monterey, CA, 2017.

[13] Johann Dambeck and Benjamin Braun. Analytical 2D GNSS PVT solutions from a hyperbolic positioning approach. GPS Solutions, 2012.

[14] Elisabeth Nossek, Johann Dambeck, and Michael Meurer. A Direct 2D Position Solution for an APNT-System. In Proc. of the Global Nav. Sat. Systems Conf. of the ION. Tampa, FL, 2015.

[15] Okuary Osechas, Nicolas Schneckenburger, Wouter Pelgrum, Elisabeth Nossek, and Michael Meurer. Characterization of the Ground-to-Air Ranging Performance of the 960-1215 MHz ARNS Band Using OFDM Measurements in the 902-928 MHz ISM Band. In Proc. of the International Technical Meeting of the ION. Monterey, $C A, 2016$. 